\title{
AMINO ACID SEQUENCE OF THE 9-kDa IRON-SULFUR PROTEIN OF PHOTOSYSTEM I IN BARLEY
}

\author{
by \\ HENRIK VIBE SCHELLER ${ }^{1)}$, IB SVENDSEN ${ }^{2)}$, and BIRGER LINDBERG MØLLER ${ }^{1)}$ \\ "Department of Plant Physiology, Royal Veterinary and Agricultural University, \\ Thorvaldsensvej 40, DK-1871 Frederiksberg C \\ and \\ 2'Department of Chemistry, Carlsberg Laboratory, \\ Gamle Carlsberg Vej 10, DK-2500 Copenhagen Valby
}

Keywords: psaC, ferredoxin, iron-sulfur center

The 9-kDa thylakoid polypeptide which in vivo carries the iron-sulfur centers $\mathrm{A}$ and $\mathrm{B}$ of photosystem I was isolated from barley (Hordeum vulgare L.) and the complete amino acid sequence determined. The polypeptide shows a very high degree of homology with the corresponding polypeptides in other plant species. The polypeptide is not post-translationally processed except for the removal of the $\mathrm{N}$-terminal formyl-methionine and the insertion of the iron-sulfur centers.

\section{INTRODUCTION}

Photosystem I contains three iron-sulfur centers: $X, A$, and $B(16,20)$. Center $X$ is bound to the reaction center protein $\mathrm{P} 700$-chlorophyll $a$-protein $1(4,9)$ while centers $\mathrm{A}$ and $\mathrm{B}$ are bound to a 9-kDa polypeptide $(10,17,18,24)$. Centers $A$ and $B$ are [4Fe-4S] clusters. One copy of the $9-\mathrm{kDa}$ polypeptide is present per $\mathrm{P} 700$ reaction center (22).

In a previous paper we have reported the isolation and partial amino acid sequence of the 9-kDa polypeptide (10). The polypeptide was identified as chloroplast encoded and the gene for the polypeptide was named psaC. The polypeptide has recently been isolated from spinach with the iron-sulfur centers more or less preserved $(17,18,24)$. The polypeptide isolated from barley contained no iron, but part of the acid-labile sulfide from the iron-sulfur clusters was retained in the form of zero-valence sulfur (10).

The amino acid sequence of the 9-kDa polypeptide has been determined for spinach (17) and deduced from the nucleotide sequence in tobacco (6), Marcantia polymorpha (19), maize (21), wheat (3), and pea (3). In the present paper we report the complete amino acid sequence of the 9-kDa polypeptide isolated from barley. The study was carried out to reveal possible post-translational modification and to permit future detailed studies on the molecular organization of photosystem I in barley.

\section{MATERIALS AND METHODS}

Chloroplast membranes, photosystem I particles, and the $9-\mathrm{kDa}$ polypeptide were isolated from barley (Hordeum vulgare cv. Svalöfs 
Bonus) as described by $\mathrm{H} ø \mathrm{~J}$ et al. (10).

Prior to cleavage reactions and sequencing, the cysteine residues in the $9-\mathrm{kDa}$ polypeptide were $S$-alkylated with 2-vinylpyridine (7). Amino acid sequences were determined with an Applied Biosystems gas-phase sequenator model $470 \mathrm{~A}$ coupled to a PTH Analyzer model $120 \mathrm{~A}$, also from Applied Biosystems (10).

Chemical cleavage at methionine residues with cyanogen bromide was carried out according to KAMP (11). The fragments obtained were separated on a column $(0.9 \times 60 \mathrm{~cm})$ of Bio-Gel P60 ( 100-200 mesh, Bio Rad Laboratories) equilibrated in $30 \%$ acetic acid.

Enzymatic cleavage of the 9-kDa polypeptide at glutamate residues was carried out with Staphylococcus aureus, strain V8, protease (11). The mixture of fragments was lyophilized, dissolved in a small volume of $0.1 \%$ trifluoroacetic acid, and separated by reverse-phase high performance liquid chromatography on a $\mathrm{C}_{18}$ column (Vydac 218TP54, The Separations Group, Hesperia, CA). Elution was carried out in the presence of $0.1 \%$ trifluoroacetic acid using a linear gradient from 0 to $54 \%$ acetonitrile.

For analysis of the C-terminal amino acid sequence (15), the $S$-alkylated polypeptide (13 $\mathrm{nmol}$ ) was incubated at room temperature with carboxypeptidase $\mathrm{Y}(0.65 \mu \mathrm{g})$. Norleucine $(14$ $\mathrm{nmol}$ ) was added as an internal standard.
Aliquots were withdrawn after different incubation periods, acidified to stop the reaction $(\mathrm{pH}$ 3.0 ), and analyzed without further treatment in a Durrum D500 amino acid analyzer.

\section{RESUITS AND DISCUSSION}

The amino acid sequence of the 9-kDa polypeptide is shown in Figure 1. Sequencing of the intact 9-kDa polypeptide enabled the determination of 29 residues. Sequencing of a cyanogen bromide fragment starting with residue 28 enabled the determination of the sequence up to residue 58 . The C-terminal cyanogen bromide fragment was not recovered from the Bio-Gel P60 column, but the sequence (Ala- 77 to Tyr-80) was determined by sequencing the total mixture of cyanogen bromide fragments. Sequencing of fragments from the cleavage reaction with $S$. aureus V8 protease resulted in the determination of the remaining sequence (Ser-55 to Glu-71 and Thr-72 to Tyr80 ). Although overlap was not established between residue 71 and residue 72 , the homology with the sequences from other species leaves no doubt about the relative position of the fragments. The carboxypeptidase $\mathrm{Y}$ digestion released successively the C-terminal residues (-SM-A-L-S-Y). The molecular mass of the reduced apoprotein is calculated to be $8768 \mathrm{Da}$. The holoprotein with the two [4Fe-4S] clusters

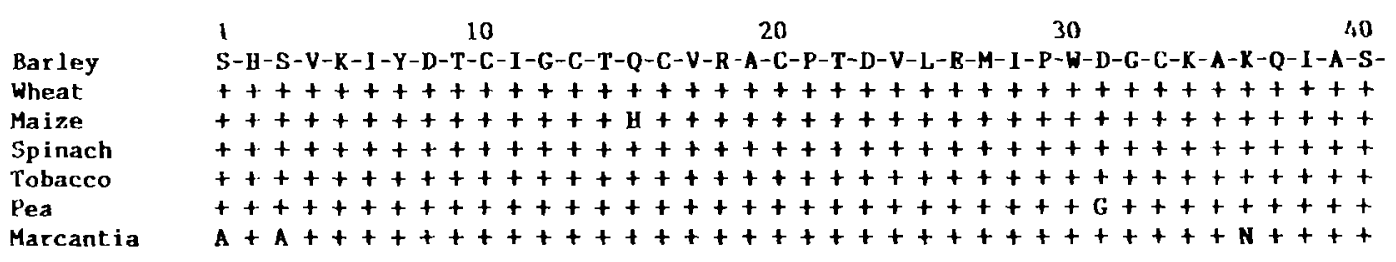

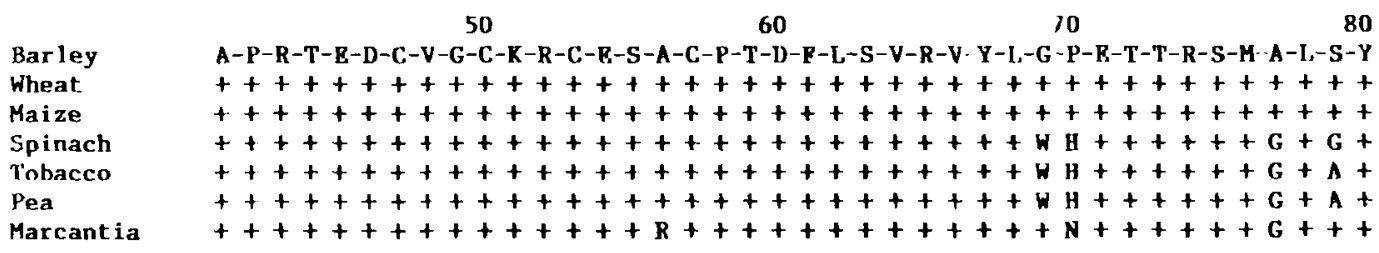

Figure 1. Amino acid sequence of the $9-\mathrm{kDa}$ polypeptide from barley. The sequence from spinach (17) as well as the sequences deduced from the nucleotide sequence of the psaC gene in Marcantia polymorpha (19), tobacco (6), maize (21), pea (3), and wheat (3) are shown for comparison. Only amino acids deviating from the barley sequence are shown. 

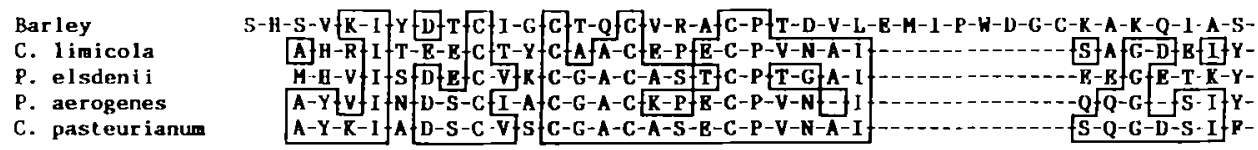

Figure 2. Comparison of the barley 9-kDa polypeptide with some bacterial ferredoxins containing two [4Fe-4S] clusters. Residues in the polypeptide from barley and the ferredoxins from Chlorobium limicola (ferredoxin II) (25), Peptostreptococcus elsdenii (25), and Peptococcus aerogenes (1), which are identical to the corresponding residues in the ferredoxin from Clostridium pasteurianum (25), are boxed. The ferredoxin from $\mathrm{C}$. pasteurianum has the most ancestral sequence (17).

is calculated to have a molecular mass of 9463 Da.

The homology between the protein from barley and the corresponding proteins from wheat, maize, tobacco, spinach, pea, and M. polymorpha is $100 \%, 99 \%, 95 \%, 95 \%, 94 \%$, and $93 \%$, respectively. The high degree of conservation of the primary structure of this protein is not solely imposed by its basic function of binding the two [4Fe-4S] clusters. Thus, bacterial 2[4Fe-4S] ferredoxins have much less homology with the 9-kDa iron-sulfur protein from photosystem I (Figure 2). OH-OKA et al. suggest that the high homology between the 9-kDa polypeptide from photosystem $I$ in different plants can be explained by a slow evolution in the more restrictive environment of a membrane-bound component as compared to a soluble protein (17). However, slow evolution is a common characteristic of chloroplast genes. Thus, the homology between the 9-kDa polypeptide in spinach and tobacco is $99 \%$, whereas the homology between the nuclear encoded subunit II of photosystem I in spinach (13) and tomato (8) is only $85 \%$. Also, the homology between soluble 2 [4Fe-4S] ferredoxins from distantly related bacteria is comparatively high (Figure 2 ). In this context it would be interesting to compare the 9-kDa polypeptide of higher plants with the corresponding membrane bound polypeptide in cyanobacteria. If membrane binding serves to increase conservation, the cyanobacterial $9-\mathrm{kDa}$ polypeptide is expected to be highly homologous to that of higher plants. A primary structure more similar to that of bacterial ferredoxins would indicate that the protein has conserved its structure due to being chloroplast encoded.

The specific binding site of the $9-\mathrm{kDa}$ polypeptide to the photosystem I complex is not known, but it involves no covalent bonds. The polarity index of the 9-kDa polypeptide calculated according to CAPALDI and VANDERKOOI (2) is $44 \%$, indicating that the polypeptide is hydrophilic compared to most membrane proteins. The polarity index for a homologous protein such as the 2[4Fe-4S] ferredoxin from Peptococcus aerogenes (1) is only $33 \%$ even though this protein is soluble. The hydrophilic nature of the 9-kDa polypeptide is evidenced by its solubility in the absence of detergents (17, 24). The 9-kDa polypeptide contains no regions that are predicted to represent membrane-spanning segments. In the cytochrome subunit of the reaction center in Rhodopseudomonas viridis, a diglyceride is covalently attached to the $\mathrm{N}$-terminal amino acid, thus providing a hydrophobic group which serves as a membrane anchor (23). A similar situation is not the case with the $9-\mathrm{kDa}$ polypeptide. The fact that the phenylthiohydantion-derivatives of all the amino acid residues could be identified strongly indicates that the 9-kDa polypeptide does not undergo post-translational processing involving covalent bonds except for the removal of the N-terminal formyl-methionine.

Comparison of the 9-kDa polypeptide to the homologous and well characterized soluble 2[4Fe-4S] ferredoxins such as the one from $P$. aerogenes (1) indicated an almost identical tertiary structure (data not shown). The differences 
in the structure are that the 9-kDa polypeptide has an extra loop with 8 residues (residues 26-33) and 14 additional residues in the C-terminal. A similar result was obtained by OH-OKA et al. with the 9-kDa polypeptide from spinach simulated on the $P$. aerogenes structure (17). The additional residues in the $\mathrm{C}$-terminal are quite hydrophilic (polarity index 50\%), and contrary to the suggestion of OH-OKA et al. (17), we do not think the C-terminal could mediate the interaction with the thylakoid membrane. Also, the C-terminal is the least conserved region of the polypeptide (see Figure 1) and therefore unlikely to have an important function. The loop formed by residues 26-33 is more hydrophobic (polarity index $25 \%$ ) and may as suggested by OH-OKA et al. (17) function as a sort of membrane anchor by which the polypeptide interacts with the thylakoid membrane or other photosystem I subunits. A hydrophobicity plot has earlier identified cysteine residues $10,13,16$, and 57 as binding center $A$ and cysteine residues 20, 47, 50 , and 53 as binding center $B(10)$. Denaturation and inhibitor studies indicate a more external location of center $B$ in the thylakoid membrane compared to center $A(5,12,14)$. The 8 residue loop extends from a region of the protein close to center A supporting the idea that this part of the protein is faced towards or embedded into the thylakoid membrane. The loop has negative charges and is therefore most likely anchored to a positive domain formed by the other photosystem I polypeptides. The charged and hydrophilic residues of the 9-kDa polypeptide are not concentrated in a certain region of the surface of the polypeptide. We suggest that the binding of the 9-kDa polypeptide to the photosystem I complex is a result of a specific interaction with residues on the reaction center protein, P700-chlorophyll $a$-protein 1 .

\section{ACKNOWIEDGEMENT}

Hanne Linde Nielsen, Inga Olsen, Bodil Corneliussen, Lone Sørensen, and Pia BredDAM are thanked for skillful technical assistance. Dr. SøREN THIRUP is thanked for performing the simulation of the structure, and Dr. PETER BORDIER HøJ is thanked for valuable discussions.

\section{REFERENCES}

1. Adman, E.T. L.C. Sieker \& L.H. Jensen: Structure of a bacterial ferredoxin. J. Biol. Chem. 248, 3987-3996 (1973)

2. Capal.DI, R.A. \& G. VanderkoOl: The low polarity of many membrane proteins. Proc. Nat. Acad. Sci. USA 69, 930-932 (1972)

3. DuNN, P.P.L. \& J.C. GRAY: Localization and nucleotide sequence of the gene for the $8 \mathrm{kDa}$ subunit of photosystem I in pea and wheat chloroplast DNA. Plant. Mol. Biol. 11, 311-319 (1988)

4. GolbeCK. J.H. \& J.M. CoRnel.ius: Photosystem I charge separation in the absence of centers $A$ and B. I. Optical characterization of center " $\mathrm{A}_{2}$ " and evidence for its association with a $64 \mathrm{kDa}$ polypeptide. Biochim. Biophys. Acta 849, 16-24 (1986)

5. GOLBFCK. J.H. \& B. KoK: Further studies of the membrane-bound iron-sulfur proteins and P700 in a photosystem I subchloroplast particle. Arch. Biochem. Biophys. 188, 233-242 (1978)

6. Hayashida. N., T. Matsubayashi. K. Shinozaki M.SLGILRA. K INOLE\& T. HIYAMA: The identification and characterization of the $9 \mathrm{kDa}$ polypeptide gene found in tobacco chloroplast DNA. Curr. Genet. 12, 247-250 (1987)

7. HeNSCHEN. A.: Analysis of cyst(e)ine residues, disulfide bridges, and sulfhydryl groups in proteins. In: Advanced Methods in Protein Microsequence Analysis. B. Wittmann-Liebold, J. Salnikow and V.A. Erdmann, eds., Springer Verlag, Berlin pp. 244-255 (1986)

8. Hoffman, N.E., F. Pichersky, V.S. Malik, K. Ko \& A.R. CASHMORE: Isolation and sequence of a tomato cDNA clone encoding subunit II of the photosystem I reaction center. Plant Mol. Biol. 10, 435-445 (1988)

9. HøJ. P.B. \& B.L. MøLLfeR: The $110 \mathrm{kDa}$ reaction center protein of photosystem I, P700-chlorophyll $a$-protein 1 , is an iron sulfur protein. J. Biol. Chem. 261, 14292-14300 (1986)

10. Høנ, P.B.. I. Svendosen. H.V. Scheller \& B.L. MøLLER: Identification of a chloroplast-encoded 9-kDa polypeptide as a $2[4 \mathrm{Fe}-4 \mathrm{~S}]$ protein carrying centers A and B of photosystem I. J. Biol. Chem. 262, 12676-12684 (1987)

11. KAMP.R.M.: Separation of peptides. In: Advanced Methods in Protein Microsequence Analysis. B. Wittmann-Liebold, J. Salnikow, and V.A. Erdmann, eds., Springer Verlag, Berlin pp. 8-20(1986)

12. Kojima, Y.. Y. NiInomi, S. Tsubol. T. Hiyama \& H. SAKURAI: Destruction of photosystem I iron-sulfur centers of spinach and Anacyctis nidulans by mercurials. Bot. Mag. (Tokyo) 100, 243-253 (1987)

13. LAGOUTTE. B.: Cloning and sequencing of spinach 
cDNA clones encoding the $20 \mathrm{kDa}$ PS I polypeptide. FEBS Lett. 232, 275-280 (1988)

14. MaLKIN,R.: Diazonium modification of photosystem I. A specific effect on iron-sulfur center B. Biochim. Biophys. Acta 764, 63-69 (1984)

15. Martin, B., I. Svendsen \& M. Ottesen: Use of carboxypeptidase $Y$ for carboxy terminal sequence determinations. Carlsberg Res. Commun. 42, 99102 (1977)

16. Møller, B.L., P.B. HøJ, B.A. Halkier, I. Olsen, H.L. Nielsen \& A. MADSEN: Photosynthesis. Dan. J. Agron. 132, 5-21 (1987)

17. OH-OKa, H., Y. Takahashi, K. Kuriyama, K. Saeki \& H. Matsubara: The protein responsible for center $\mathrm{A} / \mathrm{B}$ in spinach photosystem I: isolation with iron-sulfur cluster(s) and complete sequence analysis. J. Biochem. 103, 962-968 (1988)

18. OH-OKa, H., Y. Takahashi, H. Matsubara \& $\mathrm{S}$. ITOH: EPR studies of a $9 \mathrm{kDa}$ polypeptide with an iron-sulfur cluster(s) isolated from photosystem I complex by $n$-butanol extraction. FEBS Lett. 234, 291-294 (1988)

19. OH-OKa, H.. Y. TaKahashi, K. Wada, H. MatsubARA, K. OHYama \& H. OzeKI: The 8 kDa polypeptide in photosystem $I$ is a probable candidate of an iron-sulfur center protein coded by the chloroplast gene frxA. FEBS Lett. 218, 52-54 (1987)

20. Rutherford, A.W.\& P. HeathCote: Primary photochemistry in photosystem I. Photosynth. Res. 6, 295-316 (1985)

21. SCHANTZ, R. \& L. BOGORAD: Maize chloroplast genes ndhD, ndhE and psaC. Sequences, transcripts and transcript pools. Plant Mol. Biol. 11, 239-247 (1988)

22. SCHeller, H.V., I. SVEndSEN \& B.L. MølleR: Subunit composition of photosystem I and identification of center X as a [4Fe-4S] cluster. J. Biol. Chem. In press (1989)

23. WEYER, K.A., W. SCHÄFER, F. LOTTSPEICH \& H. Michel: The cytochrome subunit of the photosynthetic reaction center from Rhodopseudomonas viridis is a lipoprotein. Biochemistry 26 , 2909-2914 (1987)

24. WYNN, R.M. \& R. MALKIN: Characterization of an isolated chloroplast membrane $\mathrm{Fe}-\mathrm{S}$ protein and its identification as the photosystem I Fe- $\mathrm{S}_{\mathrm{A}} / \mathrm{Fe}-\mathrm{S}_{\mathrm{B}}$ binding protein. FEBS Lett. 229, 293-297 (1988)

25. Yasunobu, K.T. \& M. TANAKa: The isolation and primary sequence of various types of ferredoxin. Methods Enzymol. 69, 228-238 (1980) 